\title{
Media Information Processing of Economic Statistics and Policy Preferences During the COVID-19 Crisis* \\ Pre-Analysis Plan
}

\author{
Patrick Bareinz $^{\dagger} \quad$ Fabian Koenings ${ }^{\ddagger}$ \\ University of Jena University of Jena
}

November 14, 2020

\begin{abstract}
We investigate the effect of how media communicates information to consumers, i.e. media information processing, on policy preferences in the context of the COVID-19 crisis. For that purpose, we conduct a survey experiment to study the event of the publication of a highly regarded annual report about the state of the economy in Germany. In our experiment, individuals are exposed to information on a key economic statistic covered in the report which differs in terms of its framing. This design allows us to disentangle effects which are related to the published information in the original press release and its coverage by media outlets. Our examination accounts for public evaluation of economic policy in general as well as important subdomains. We aim to inform about policy implications on the relationship between media coverage, belief updating and public opinion formation in times of economic recession and pronounced public interest in statistical reporting.
\end{abstract}

JEL classification: C90, D83, D91, L82.

Keywords: survey exeriment, media bias, information processing, belief updating, economic policy preferences, COVID-19 crisis.

${ }^{*}$ IRB approval was obtained at FSU Jena.

'University of Jena, Carl-Zeiss-Str. 3, 07743 Jena, Germany, email: patrick.bareinz@uni-jena.de.

łUniversity of Jena, Carl-Zeiss-Str. 3, 07743 Jena, Germany, email: fabian.koenings@uni-jena.de. 


\section{Motivation}

The COVID-19 pandemic and the corresponding global economic crisis impacts economic policy in many countries. In this environment of economic recession and pronounced uncertainty, the reporting of media outlets such as newspapers is an important determinant of public opinion formation. There is a growing literature on the effects of media on political attitudes and voting behavior (Allcott and Gentzkow 2017; DellaVigna and Kaplan 2007; Gerber et al. 2009) and in the context of media bias and demand for news (Chopra et al. 2019; Gentzkow and Shapiro $2006,2010)$.

We aim to extend this literature by investigating whether the framing of statistical information by media outlets affects economic policy preferences in a setting of pronounced demand for information. Specifically, we conduct a survey experiment on media bias in the context of the COVID-19 crisis in Germany. In our experiment, we inform survey respondents about a key economic statistic covered in a highly regarded annual report about the state of the economy in Germany.

By experimentally varying the provision of original information and its media coverage, we aim to disentangle effects of how the media frames this press release material and the information itself. Besides these insights on the effect of framing by the media, this investigation is also highly relevant for a better understanding of the relationship between dissemination of statistical information and public opinion formation during a global health and economic crisis.

\section{Experimental Design}

In the following, we introduce our experimental design, consisting of four stages and four experimental groups. Survey respondents are randomly assigned to one of two treatment arms which differ with respect to the framing of the information provided, to the active control group receiving the original information, or to the passive control group which does not receive any information.

The information which is provided to respondents stems from a highly regarded report about the state of the German economy by the German Council of Economic Experts (GCEE). The annual report is presented to the public regularly in November and includes a press release 
pointing out key information from the comprehensive report. We employ both the original press release as well as the media coverage in German news outlets which is generated by the publication of the GCEE report on an annual basis.

\subsection{Elicitation of prior beliefs}

First stage:

- Elicitation of respondents' beliefs about a key economic statistic covered in the GCEE report, i.e. the forecasted GDP growth rate for 2021

\subsection{Treatment and control groups}

Second stage:

- Random subsets of respondents are exposed to differently framed information on the forecasted GDP growth rate for 2021

- Treatment arm I: receives positively framed information from the GCEE press release as covered by a large German online news outlet

- Treatment arm II: receives negatively framed information from the GCEE press release as covered by another large German online news outlet

- Control group I (active): receives the original information based on the GCEE press release

- Control group II (passive): does not receive any information

Our experimental design enables us to differentiate between the "pure" effect of providing the original information as stated in GCEE press release and the effects of framing the original information by media outlets.

\subsection{Outcome variables}

Third stage:

- Respondents are asked about their evaluation and preferences with respect to the general COVID-19 policy as well as important subdomains of economic policy: 
- Labor market policy

- Health policy

- Education policy

All outcome measures are measured on an 11-point scale.

\subsection{Elicitation of posterior beliefs}

Fourth stage:

- Elicitation of posterior beliefs about the forecasted GDP growth rate for 2021 for those respondents who did receive information on the topic

To mitigate concerns about experimenter demand, we elicit posterior beliefs at the final stage of the survey. The elicitation of posterior beliefs allows us to investigate whether respondents update their beliefs and how the framing of information contributes to potential differences in belief updating.

\section{Main Hypotheses}

In the following, we derive our main hypotheses for our information provision experiment: Hypothesis I: Overestimation:

Revealed overestimation of the forecasted GDP growth rate for 2021 leads to a more negative evaluation of policies and less supportive preferences.

Hypothesis II: Underestimation:

Revealed underestimation of the forecasted GDP growth rate for 2021 leads to a more positive evaluation of policies and more supportive preferences.

Hypothesis III: Positive framing:

Positive framing of the forecasted GDP growth rate for 2021 leads to a more positive evaluation of policies and more supportive preferences. 
Hypothesis IV: Negative framing:

Negative framing of the forecasted GDP growth rate for 2021 leads to a more negative evaluation of policies and less supportive preferences.

The structure of the survey experiment allows for the hypotheses to amplify and offset each other. In addition, the investigation of belief updating in the several contexts will be subject to further heterogeneity analyses.

\section{Data}

We embed our survey experiment into a large-scale representative online survey of 3000 individuals in Germany. The survey is representative with respect to age, gender, educational background, and place of residence in Eastern/Western Germany. The field phase starts in November 2020 and the survey will be distributed to respondents by a professional survey company via an online panel.

The survey contains measures about the assessment of the current economic situation, media consumption, beliefs about the forecasted GDP growth rate for 2021, assessment of COVID-19 policies, policy preferences, concerns about the COVID-19 crisis, and general political and social attitudes.

\section{Analysis}

The following section describes our empirical approach to the investigation of treatment effects and belief updating of respondents.

\subsection{Experimental balance}

We start by conducting tests for experimental balance between control and treatment groups, based on between-subject t-tests. An alternative approach to the assessment of experimental balance in case of large samples are normalized differences, as put forward by Imbens (2015). The following list contains the covariates we plan to include in the balance tests:

- prior beliefs about the forecasted GDP growth rate for 2021 
- concerns about the economic situation

- risk and trust attitudes

- media consumption

- political attitude

- financial deficits due to the COVID-19 crisis

- contact with COVID-19 infected person

- age group

- gender

- residence

- education

- employment status

- household net income

- household size

- relationship status

- migration background

- rural area of residence

\subsection{Updating of prior beliefs and heterogeneous updating of treatment groups}

We conduct within-subject t-tests between prior and posterior beliefs in the treatment groups and the active control group to examine belief updating of respondents.

We also investigate whether there are differences in belief updating across these experimental groups which receive differently framed information. For that purpose, the following equation is estimated:

$$
u_{i}=\delta_{0}+\delta_{1} T_{i}^{I}+\delta_{2} T_{i}^{I I}+\varepsilon_{i}
$$


where $u_{i}$ represents belief updating of respondents (i.e. the within-subject difference between posterior and prior beliefs), $T_{i}^{I}$ and $T_{i}^{I I}$ are treatment indicators for the two treatment groups, and $\varepsilon_{i}$ is the error term. Since there are no posterior beliefs for the passive control group which does not receive any information, this specification is only estimated for the two treatment groups and the active control group. Hence, the active control group serves as the base group.

\subsection{Global effects of information}

We estimate the following equation to examine global, i.e. full-sample effects of information provision, comparing our outcome variables across experimental groups given their exogeneity:

$$
y_{i}=\gamma_{0}+\gamma_{1} T_{i}^{I}+\gamma_{2} T_{i}^{I I}+\gamma_{3} C_{i}^{I}+\varepsilon_{i}
$$

where $y_{i}$ represents the outcome variable, $T_{i}^{I}$ and $T_{i}^{I I}$ are treatment indicators for the two treatment groups, $C_{i}^{I}$ is an indicator for the active control group, and $\varepsilon_{i}$ is the error term.

In order to disentangle the framing from the general information effect, we furthermore estimate the following equations for the active control group using the passive control group as the base group:

$$
y_{i}=\alpha_{0}+\alpha_{1} C_{i}^{I}+\varepsilon_{i}
$$

and for both treatments groups using the active control group as the base group:

$$
y_{i}=\beta_{0}+\beta_{1} T_{i}^{I}+\beta_{2} T_{i}^{I I}+\varepsilon_{i}
$$

respectively, where $y_{i}$ represents the outcome variable, $T_{i}^{I}$ and $T_{i}^{I I}$ are treatment indicators for the two treatment groups, $C_{i}^{I}$ is an indicator for the active control group, and $\varepsilon_{i}$ is the error term.

\subsection{Heterogeneity in treatment effects}

In addition to the evaluation of global effects of information provision, we analyze potential differences in treatment responsiveness across subgroups of the population. Following a systematic data-driven approach developed by Athey and Imbens $(2016,2019)$ called causal tree analysis, we aim to uncover potential heterogeneity in treatment effects. 
By means of a recursive approach, this machine learning algorithm sequentially partitions the data into a structure of subsamples. These subsamples are constructed based on the mean-squared error (MSE) of the conditional average treatment effect (CATE) (Athey and Imbens 2016). This procedure generates a visual representation of sequential treatment effect heterogeneity, i.e the causal tree. In our context, the algorithm is supplied with the covariates from the balance tests and is trained to evaluate subgroups of at least 50 respondents.

We will then estimate the specifications for the information and framing investigations again for the most relevant subgroups on the basis of CATE. Furthermore, we plan to investigate heterogeneity with respect to media consumption and preferences, as well as individual concerns about the COVID-19 crisis.

\subsection{Determinants of biases in prior beliefs}

We also explore the association of covariates with biased beliefs about the forecasted GDP growth rate for 2021. Specifically, we estimate the following equation:

$$
b_{i}=\theta_{0}+\theta^{\prime} X_{i}+\varepsilon_{i}
$$

where $b_{i}$ represents biases in beliefs about the key economic statistic, $X_{i}$ contains the sociodemographic and attitudinal controls used in the balance tests, and $\varepsilon_{i}$ is the error term.

\subsection{Further strategies for analysis}

In the following, we discuss further strategies for our empirical analysis.

\subsubsection{Adressing potential imbalances}

It may be that, despite randomization, there exist imbalances between experimental groups for some covariates. In our estimation specifications, we will therefore control for these imbalances in terms of observables. 


\subsubsection{Pooling of experimental groups}

Since our two treatment arms are both related to framing of information, we consider a pooling strategy for these two experimental groups. In addition, a pooling strategy for the three groups which received information against the passive control group is considered.

\subsubsection{Indices}

Since our outcome measures are related, they allow for the construction of indice measures, e.g. an additive index of the three subdomains of policy preferences. 


\section{References}

Allcott, Hunt and Matthew Gentzkow (2017). "Social Media and Fake News in the 2016 Election". Journal of Economic Perspectives 31 (2), 211-236.

Athey, Susan and Guido W. Imbens (2016). "Recursive Partitioning for Heterogeneous Causal Effects". Proceedings of the National Academy of Sciences 113 (27), 7353-7360.

- (2019). “Machine Learning Methods That Economists Should Know About". Annual Review of Economics 11 (1), 685-725.

Chopra, Felix, Ingar Haaland, and Christopher Roth (2019). “Do People Value More Informative News?" mimeo.

DellaVigna, Stefano and Ethan Kaplan (2007). "The Fox News Effect: Media Bias and Voting". The Quarterly Journal of Economics 122 (3), 1187-1234.

Gentzkow, Matthew and Jesse M. Shapiro (2006). "Media Bias and Reputation". Journal of Political Economy 114 (2), 280-316.

— (2010). "What Drives Media Slant? Evidence From U.S. Daily Newspapers". Econometrica $78(1), 35-71$.

Gerber, Alan S., Dean Karlan, and Daniel Bergan (2009). “Does the Media Matter? A Field Experiment Measuring the Effect of Newspapers on Voting Behavior and Political Opinions". American Economic Journal: Applied Economics 1 (2), 35-52.

Imbens, Guido W. (2015). “Matching Methods in Practice: Three Examples". Journal of Human Resources 50 (2), 373-419. 